\title{
In situ transmission electron energy loss spectroscopy of e-beam modifications in oxide glasses and crystals
}

\author{
Nan Jiang \\ Department of Physics and Astronomy, Arizona State University, Tempe AZ 85287-1504 \\ Electrostatic field-enhanced migration of ions in oxide glasses and crystals has been \\ intensively observed in scanning transmission electron microscopy (STEM) [1]. In this case, \\ excitation by fast electrons causes that some electrons in the atoms are driven out of the \\ trajectory of the beam. The trajectory region thus becomes charged positively and positive \\ ions are repelled from its vicinity [2]. The dynamics of ion migration can be directly \\ observed using in situ electron energy loss spectroscopy (EELS) technique, which has been \\ able to reveal a wealth of information about electronic and atomic structure, and chemistry of \\ interaction region [3]. In contrast to its influential role in exploring microstructures of \\ crystalline materials, electron microscopy has little impact on understanding of amorphous \\ materials. Recently, EELS technique has been applied to the study of long-range structural \\ fluctuation in glasses [4]. In this work, we demonstrate that in situ EELS technique can also \\ play an important role in the study of glass structures.
}

Fig. 1 shows the in situ EELS of O K-edge in crystalline $\mathrm{CaAl}_{2} \mathrm{O}_{4}$. The sharp peak at about $528 \mathrm{eV}$ is from $\mathrm{O}_{2}$, which is induced during electron irradiation. According to electronic structure calculations (not shown here), the broad peak between 530 and $535 \mathrm{eV}$ is mainly due to $\mathrm{Ca}-\mathrm{O}$ interaction, while feature between 535 and 540 is mainly due to $\mathrm{Al}-\mathrm{O}$ interaction. The intensity profile of $\mathrm{O}_{2}$ peak, as well as $\mathrm{Ca}_{23}$-edge, is also given in Fig. 1. It is noted that the phenomena of ion migrations shown in Fig. 1 are the exactly same with the observations in other glasses (e.g. $\mathrm{CaO}-\mathrm{Al}_{2} \mathrm{O}_{3}-\mathrm{SiO}_{2}, \mathrm{~K}_{2} \mathrm{O}-\mathrm{SiO}_{2}$ and $\mathrm{ZnO}-\mathrm{B}_{2} \mathrm{O}_{3}-\mathrm{SiO}_{2}$ ). The details of dynamics have been discussed in [3]. Here two additional features need to be pointed out. One is that nearly half amount of $\mathrm{Ca}$ still remains in the illuminated region after rearrangement of $\mathrm{O}$ ceases (i.e. the change of $\mathrm{O} \mathrm{K}$-edge stops). The question is: why do some $\mathrm{Ca}$ ions prefer to be repelled by electrostatic field but others not? The other is that the threshold of $\mathrm{O} \mathrm{K}$-edge shifts upwards about $0.5 \mathrm{eV}$. The thresholds of the $\mathrm{O} \mathrm{K}$-edge are about 530.8 in the initial spectrum and $531.3 \mathrm{eV}$ after $190 \mathrm{~s}$ of irradiation. The intensity profile of $\mathrm{O}$ $\mathrm{K}$-edge in the threshold region (integrated from 530 to $532 \mathrm{eV}$ ) is compared with the profile of $\mathrm{Ca}$ in Fig. 1. Interestingly, the threshold intensity drops significantly within about 70 s of irradiation, and becomes relatively constant thereafter. At this point, the irradiated region loses about one third of $\mathrm{Ca}$.

In $\mathrm{CaAl}_{2} \mathrm{O}_{4}$, two third $\mathrm{Ca}$ is 6 -fold coordination $\mathrm{Ca}(6)$ and one third is 9-fold $\mathrm{Ca}(9)$. Correspondingly, $\mathrm{O}$ can also be categorized into three groups: $\mathrm{O}[\mathrm{Ca}(6)]$ is bound to $\mathrm{Ca}(6)$ only, $\mathrm{O}[\mathrm{Ca}(9)]$ is bound to $\mathrm{Ca}(9)$ only and the others are bound to both. (Each $\mathrm{O}$ atom is also bound to two Al.) Fig. 2 shows the calculations of density of states (DOS) projected on two different types of $\mathrm{Ca}$ and $\mathrm{O}$. It is seen that the threshold of the total DOS on $\mathrm{Ca}(9)$ is about $0.5 \mathrm{eV}$ lower than that on $\mathrm{Ca}(6)$. Correspondingly, the threshold of $\mathrm{O} p$-DOS on $\mathrm{O}[\mathrm{Ca}(9)]$ is also about $0.5 \mathrm{eV}$ lower than that on $\mathrm{O}[\mathrm{Ca}(6)]$. Therefore, we can assign the threshold 
intensity of O K-edge (530 532eV) to the oxygen that is bound to 9-fold coordinated Ca. Eliminating this type of oxygen is associated with the repulsion of $\mathrm{Ca}(9)$ by electrostatic field. In conclusion, the 9-fold $\mathrm{Ca}$ can be repelled more easily than the 6-fold $\mathrm{Ca}$. This criterion can be extended to glasses to identify cation coordination, which is crucial to the measurement of medium-range order in glasses.

\section{References}

[1] N. Jiang, et al., Appl. Phys. Lett. 77, 3956 (2000); N. Jiang, et al., Appl. Phys. Lett. 80, 2005 (2002); N. Jiang, et al., Appl. Phys. Lett. 83, 551 (2003).

[2] N. Jiang and J. Silcox, J. Appl. Phys. 92, 2310 (2002).

[3] N. Jiang, et al., Phys. Rev. B 68, 064207 (2003).

[4] N. Jiang, et al., Phys. Rev. B 66, 054203 (2002).

[5] This work is supported by the NSF Grant No. DMR-0245702.
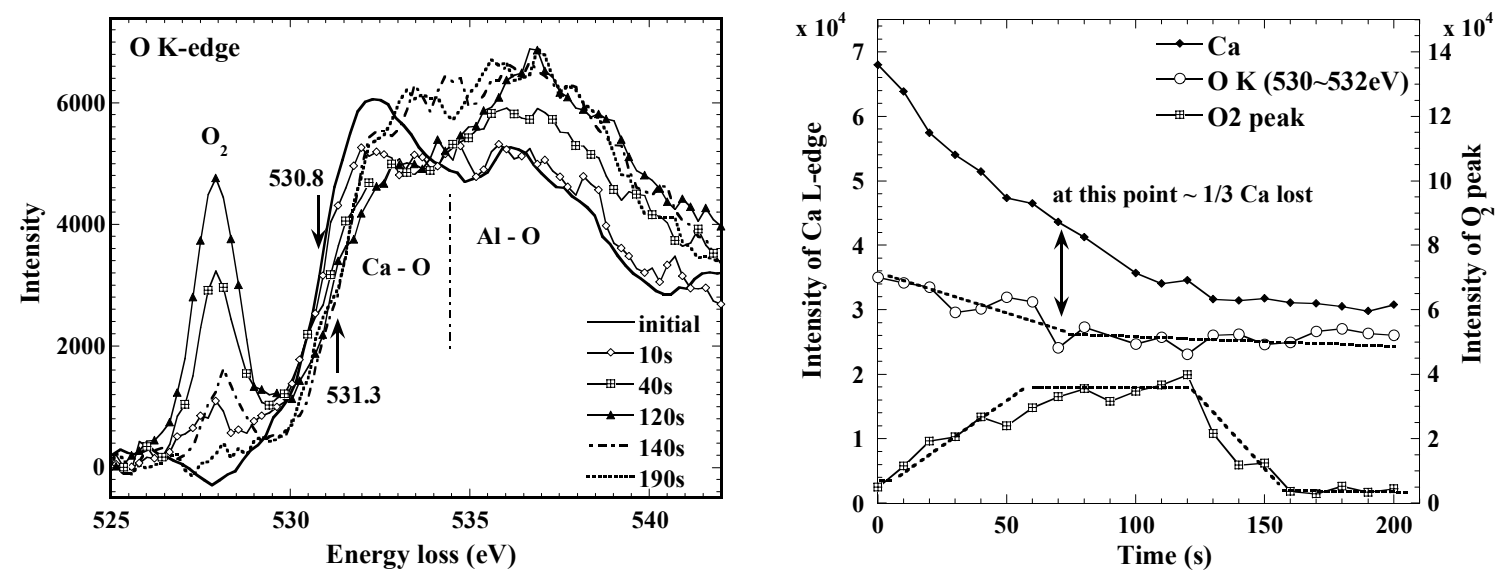

Fig. 1 In situ EELS of O K-edge using STEM (left) and EELS intensity profiles (right). Energy resolution is about $0.8 \mathrm{eV}$. The arrows in the left indicate the thresholds of the $\mathrm{O} \mathrm{K}$ edge and in the right indicate the point that the drop of threshold intensity stops. The dotted lines are guide for eyes.

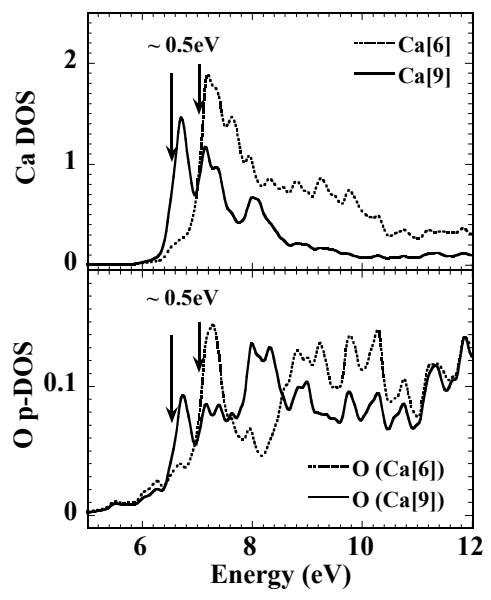

Fig. 2 Calculations of the total DOS projected on $\mathrm{Ca}$ and $\mathrm{p}$-DOS on $\mathrm{O}$ in $\mathrm{CaAl}_{2} \mathrm{O}_{4}$ using Wien code. The experimental lattice parameters atom positions are used. Other parameters in the calculations are $\mathrm{K}_{\max } \cdot \mathrm{R}_{\mathrm{MT}}=6$ and $56 \mathrm{k}$-points selected. 